\title{
ORTHOTOPIC HEART TRANSPLANTATION FOR :KAWASAKI DISEASE AFTER RUPTURE OF A GIANT CORONARY ARTERY ANEURYSM
}

Theodore C. Koutlas, MD, ${ }^{a}$ Gil Wernovsky, MD, ${ }^{\mathrm{b}}$ Nancy D. Bridges, MD, ${ }^{\mathrm{b}}$ Elsa J. Suh, MD, ${ }^{\mathrm{b}}$ Rodolfo I. Godinez, MD, ${ }^{\mathrm{c}}$ Susan C. Nicolson, MD, ${ }^{\mathrm{c}}$ Thomas L. Spray, MD, ${ }^{\mathrm{a}}$ and J. William Gaynor, MD, ${ }^{\mathrm{a}}$ Philadelphia, Pa.

Kawasaki disease $(\mathrm{KD})$ is an uncommon multisystem inflammatory disease of children in which the cardiovascular system is involved with a nonspecific vasculitis of the major branches of the aorta including the coronary arteries. Coronary artery aneurysms (CAAs) occur in $20 \%$ of untreated patients and may result in acute thrombosis or progressive stenosis of the coronary artery. ${ }^{1}$ We describe a case of ruptured giant CAA of the left main coronary artery (LMCA) treated by emergency coronary artery bypass grafting, postoperative extracorporeal membrane oxygenation, and subsequent orthotopic cardiac transplantation.

Case report. A 3-year-old white boy was transferred to the Children's Hospital of Philadelphia after 2 weeks of fever, malaise, and poor oral intake. His physical examination showed conjunctivitis, an erythematous oropharynx, and cervical lymph node enlargement. Laboratory findings included a white blood cell count of 20,000 cells $/ \mu \mathrm{l}$, hematocrit of $27 \%$, a platelet count of 835,000 cells $/ \mu \mathrm{l}$, and an erythrocyte sedimentation rate of 75 $\mathrm{mm} / \mathrm{hr}$. The electrocardiogram was normal, and the chest radiograph demonstrated small pleural effusions. A twodimensional echocardiogram revealed a $5 \mathrm{~mm}$ aneurysm of the right coronary artery and a "giant" $(2.5 \mathrm{~cm}$ long and $1.5 \mathrm{~cm}$ in diameter) aneurysm of the LMCA. Left ventricular function was normal, and there was no mitral regurgitation. Treatment for $\mathrm{KD}$ was begun with aspirin and a $2 \mathrm{gm} / \mathrm{kg}$ dose of intravenous gammaglobulin ( $\mathrm{IgG}$ ).

On hospital day 2, the patient had a cardiac arrest. Electrocardiography revealed electromechanical dissociation; cardiopulmonary resuscitation was initiated, ice was placed on the patient's head, and endotracheal intubation was performed. A stable blood pressure was obtained, and an echocardiogram demonstrated a tense hemopericardium. He was transferred to the operating room, where, after median sternotomy and decompression of the hemo-

From the Division of Pediatric Cardiothoracic Surgery, ${ }^{a}$ Division of Cardiology, ${ }^{\mathrm{b}}$ Department of Anesthesiology, The Children's Hospital of Philadelphia, Philadelphia, Pa.

Received for publication June 21, 1996; accepted for publication August 8, 1996.

Address for reprints: J. William Gaynor, MD, Pediatric Cardiothoracic Surgery, The Children's Hospital of Philadelphia, 34th St. \& Civic Ctr. Blvd., Philadelphia, PA 19104.

J Thorac Cardiovasc Surg 1997;113:217-8

Copyright (C) 1997 by Mosby-Year Book, Inc.

$0022-5223 / 97 \$ 5.00+0 \quad \mathbf{1 2 / 5 4 / 7 7 1 8 4}$ pericardium, the LMCA aneurysm was found to have ruptured. The patient was placed on cardiopulmonary bypass, and coronary artery bypass grafting was performed with an in situ graft of the left internal thoracic artery to the left anterior descending artery and a saphenous vein graft to a large circumflex marginal branch. The LMCA aneurysm was then oversewn. The patient was weaned from cardiopulmonary bypass on inotropic support consisting of epinephrine, dopamine, and amrinone infusions. The electrocardiogram 6 hours after cardiac arrest demonstrated a large anterolateral infarct. During the first postoperative night, the patient had recurrent ventricular tachycardia despite a lidocaine infusion and required DC cardioversion on several occasions. Because of progressive ventricular dysfunction and recurrent ventricular dysrhythmias, he was placed on venoarterial extracorporeal membrane oxygenation the following morning. Transesophageal echocardiography revealed a dilated left ventricle with markedly diminished shortening as a result of akinesis of the septal and anterior walls. The patient was noted to be neurologically intact after emerging from anesthesia, and after discussion with the family he was listed for heart transplantation.

$\mathrm{He}$ remained in stable condition on extracorporeal membrane oxygenation without evidence of end-organ dysfunction, but there was no improvement in his cardiac function, as assessed by serial transesophageal echocardiography. On postoperative day 4 , he underwent a successful orthotopic heart transplant; he was weaned from mechanical ventilation and inotropic support within 2 days. Immunosuppression (cyclosporine, azathioprine, and prednisone) was maintained after operation according to our standard protocol. His postoperative neurologic examination was notable only for a mild generalized weakness. The first week after transplantation, fever and a moderate increase in erythrocyte sedimentation rate developed. He was treated empirically for recurrent $\mathrm{KD}$ with a 4-day course of intravenous $\operatorname{IgG}(2 \mathrm{gm} / \mathrm{kg})$ and aspirin, with normalization of his temperature and erythrocyte sedimentation rate. Cardiac catheterization on posttransplant day 14 showed a normal cardiac index and a normal mean pulmonary capillary wedge pressure. Results of coronary angiography were also normal, and an endomyocardial biopsy specimen revealed class $1 \mathrm{~A}$ rejection. The patient was discharged home on posttransplant day 18 .

$\mathrm{He}$ has done well, with a rapid increase in his level of activity to that of a healthy child of his age. An endomyocardial biopsy performed 2 months after transplantation revealed grade 3 rejection, and he was treated with intravenous corticosteroids, with improvement to grade 2 rejection. Tacrolimus (FK 506) was then substituted for 
cyclosporine, and a subsequent endomyocardial biopsy sample showed complete resolution of the rejection. He is currently well 10 months after transplantation.

Discussion. CAAs may be detected as early as 6 days after the onset of symptoms of $\mathrm{KD}$, but peak detection is around 2 weeks into the illness. More than $50 \%$ of patients with documented CAA show resolution within 1 year of diagnosis, and $80 \%$ of small to moderate-sized aneurysms regress within 5 years of the disease. ${ }^{1} \mathrm{~A}$ significant reduction in the incidence of CAA was demonstrated in a randomized study comparing aspirin plus IgG therapy with aspirin alone in the treatment of $\mathrm{KD}^{2}$ Giant (>8 mm diameter) CAAs occur in only $5 \%$ of patients with KD, but they usually show little or no regression with time. More than half of these patients eventually have occlusive disease, and as many as $40 \%$ of giant CAAs eventually lead to myocardial infarction. $^{1}$

Rupture of giant CAA is a rare complication of $\mathrm{KD}$. In nationwide surveys in Japan, 14 cases of ruptured aneurysms have been reported, with no survivors (Takahashi M, personal communication). Four cases have been reported in the European literature, also without survivors. $^{3-5}$ Rupture of CAAs occurs during the acute phase of $\mathrm{KD}$, at the peak of the vasculitis and before intimal proliferation has begun. Each of the previously described cases of rupture occurred before the widespread use of IgG.

Orthotopic heart transplantation has been reported for 11 patients with $\mathrm{KD}{ }^{6}$ The most common indication for transplantation was ischemic left ventricular dysfunction, usually later than 1 year after KD. There have been two deaths among the published cases, one of severe rejection 10 months after transplantation and another of sequelae of long-term cardiac dysfunction. Our patient was the first to undergo heart transplantation during the acute phase of KD. Among our theoretic concerns with respect to this mode of treatment were that the heightened immune response associated with acute $\mathrm{KD}$ would lead to early rejection of the graft and that sequelae of $\mathrm{KD}$ might develop in the donor heart. Neither of these potential problems occurred during the posttransplantation course of our patient.

\section{REFERENCES}

1. Gersony WM. Diagnosis and management of Kawasaki disease. JAMA 1991;265:2699-703.

2. Newburger JV, Takahashi M, Burns JC, et al. The treatment of Kawasaki syndrome with intravenous gammaglobulin. N Engl J Med 1986;315:341-7.

3. Laane BF. Infantile polyarteritis nodosa or mucocutaneous lymph node syndrome (Kawasaki disease): arteritis associated with aneurysm, thromboses, and rupture of the coronary artery with cardiac tamponade in a 31/2-month-old boy. Tidsskr Nor Laegefore 1981;101:1583-5.

4. Lunkenheimer A, Mellin W, Pfefferkorn J, et al. Rupture of a coronary artery aneurysm in mucocutaneous lymph node syndrome (Kawasaki syndrome): demonstration of the coronary aneurysms by 2-dimensional echocardiography. Monatsschrift Kinderheilkunde 1984;132:909-12.

5. Shiller B, Fasth A, Björkhem, et al. Kawasaki disease in Sweden: incidence and clinical features. Acta Paediatr 1995; 84:769-74.

6. Checcia P. In: Kato $H$, editor. Proceedings of the fifth international Kawasaki disease symposium, Fukuoka, Japan: Elsevier, 1995:522-6. 